\title{
Coordinate-free Formulation of a 3-2-1 Wire-based Tracking Device using Cayley-Menger Determinants
}

\author{
Federico Thomas $\dagger$, Erika Ottaviano $\neq$, Lluis Ros $\dagger$, and Marco Ceccarelli \\ †Institut de Robòtica i Informàtica Industrial (CSIC - UPC) \\ Llorens Artigas 4-6, 2 planta, 08028 Barcelona, Spain \\ \{fthomas, llros\}@iri.upc.es \\ \$ Laboratorio de Robotica e Meccatronica \\ Università degli Studi di Cassino \\ Via G. Di Biasio, 43, 03043 Cassino, Italy \\ \{ottaviano, ceccarelli\}@unicas.it
}

\begin{abstract}
This paper deals with the problem of estimating the pose of a rigid moving object by measuring the length of six wires attached to it. Among all possible locations for the attachments on the moving object, the "3-2-1" configuration exhibits the highest number of favorable properties. A closed-form coordinate-free solution to the forward kinematics of this particular configuration is given in terms of Cayley-Menger determinants. The proposed formulation is mathematically more tractable compared to previous ones because all terms are determinants with geometric meaning. This accommodates a more thorough investigation of the properties of the device and leads to formulas whose numerical conditioning is independent from the chosen reference frames.
\end{abstract}

\section{INTRODUCTION}

Many systems for measuring the pose, i.e. position and orientation, of moving objects, also known as tracking systems, have been developed. They can be classified according to the measuring principle and used technology. Most systems provide distance measurements by triangulation or trilateration techniques. Trilateration and triangulation determine the relative position between points by using the geometry of triangles or tetrahedra. Triangulation uses measurements of both distances and angles, whereas trilateration uses only distance measurements.

Tracking systems can also be classified according to their characteristics, such as accuracy, resolution, cost, measurement range, portability, and calibration requirements. Laser tracking systems exhibit good accuracy, which can be less than $1 \mu \mathrm{m}$ if the system is well calibrated. Unfortunately, they are very expensive, their calibration procedure is time consuming, and they are sensitive to the environment. Vision systems have an accuracy of $0.1 \mathrm{~mm}$, they are low cost portable devices but their calibration procedure can be complicate. Wire-based systems have an accuracy of $0.1 \mathrm{~mm}$,

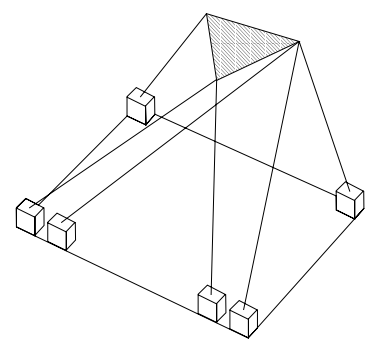

(a)

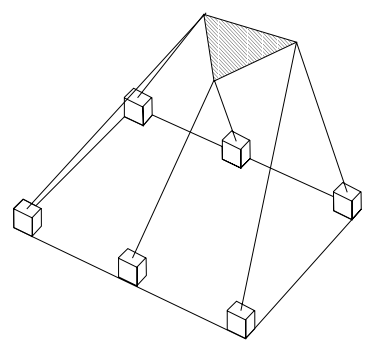

(b)
Fig. 1. (a) The "3-2-1" and (b) "2-2-2" configurations for wire-based tracking devices as proposed in [6] and [10], respectively.

they are also low cost portable devices but capable of measuring large displacements. Moreover, they exhibit a good compromise among accuracy, measurement range, cost and operability.

Wire-based tracking devices consist of a fixed base and a platform connected by six wires whose tension is maintained, while the platform is moved, by pulleys and spiral springs on the base, where a set of encoders give the length of the wires. They can be modelled as six-degree-of-freedom parallel manipulators because wires can be seen as extensible legs connecting the platform and the base by means of spherical and universal joints, respectively.

Dimension deviations due to fabrication tolerances, wire length uncertainties, wire slackness, etc., may result in an unacceptable performance of a wire-based tracking device. In general, the effects of all systematic errors can be eliminated by calibration. Some techniques for specific errors have already been proposed in the literature. For example, a method for compensating the cable guide outlet shape of wire encoders is detailed in [6], and a method for compensating the deflections caused by wire self-weights is described in [10]. In this 
paper, we will only consider wire length errors which cannot be compensated because of their random nature.

An important issue in wire-based tracking devices is the number of attachments on the moving object and how many wires are connected to each attachment. On the base side, no two wire outlets can be made coincident because of physical limitations. This is not the case on the platform where it is advisable to reduce the number of attachments not only for simplicity reasons but also to reduce the risk of wire collisions -if all attachments would collapse into a single point on the platform, no collisions would be possible. Unfortunately, the minimum number of points for pose measurements is three. Moreover, the maximum number of wires attached to a point is three, otherwise the lengths of the wires will not be independent. This leads to only two possible configurations for the attachments on the moving object. Both have already been reported in the literature of wire-based tracking devices.

- The 2-2-2 configuration. This configuration was first proposed in [10] for a wire-based tracking device [see Figure 1(a)]. The authors overlooked the fact that the kinematics of this configuration was already studied, for example, in [7], [15], and [18] where it was shown that its forward kinematics has 16 solutions. In other words, there are up to 16 poses for the moving object compatible with a given set of wire lengths. These configurations can only be obtained by a numerical method, which limits the use of this configuration in real-time measurements.

- The 3-2-1 configuration. This configuration was proposed in [6] [see Figure 1(b)]. The authors present it as a new configuration of a Stewart platform thus ignoring that the kinematics of this configuration was already studied, for example, in [14] and [9]. Its direct kinematics can be solved in closed-form by using three consecutive trilateration operations yielding 8 solutions, which is the minimum number of solutions for the direct kinematics of a 6 degree of freedom parallel platform.

Since, due to physical limitations, wire-based tracking devices work only in one of the two half-spaces defined by the base plane, the number of solutions for both configurations are, in practice, 8 and 4 , respectively. As a consequence, in order to avoid ambiguities, it is preferable to work with the 3-2-1 configuration. In this paper, we concentrate our efforts on this configuration because of this reason.

Both configurations were compared in [6] in terms of their sensitivity to wire length errors concluding that they have similar properties. Nevertheless, the used sensitivity index has important drawbacks as it depends on arbitrary choices. This sensitivity analysis is reviewed in Section II which motivates the search for a coordinate-free formulation for the forward kinematics of the 3-2-1 configuration.

All operations for solving the direct kinematics of the 3-2-1 configuration rely on trilateration. Although the trilateration problem can be trivially expressed as the problem of finding the intersection of three spheres, different closed-form solutions have been proposed in the areas of computer graphics [5], robotics [2], and aeronautics [13]. Section III presents some basic properties of Cayley-Menger determinants related to the geometry of tetrahedra which are the key elements for the new vectorial coordinate-free solution to the trilateration problem presented in Section IV. Based on this new formulation, a complete error analysis for trilateration, including covariances and bias errors of the estimations is also given in this section. Next, the forward kinematics and the singularities of the 3-2-1 configuration are analyzed in Section V. Finally, we conclude in Section VI.

\section{SENSITIVITY TO WIRE LENGTH ERRORS}

In what follows we will assume that the measured wire lengths are corrupted by random noise with gaussian probability density function.

In order to describe the relative position between base and platform, let us introduce an absolute and a relative frame fixed to the base and the platform, respectively. The pose of the platform with reference to the base is given by the configuration vector $\mathbf{q}=$ $(\omega, \mathbf{t})$, where $\mathbf{t}$ stands for the translation vector and $\omega$ for the orientation vector given in roll, pitch and yaw angles, i.e. $\mathbf{q} \in \mathfrak{R}^{3} \times S O(3)$. The corresponding rotation matrix will be denoted $\mathbf{R}(\omega)$. The unit vector along wire $i$ will be denoted $\mathbf{e}_{i}$, and $l_{i}$ the corresponding wire length. The centers of the articulations on the base and the platform for wire $i$ will be denoted $\mathbf{a}_{i}$ and $\mathbf{b}_{i}$, respectively. According to these definitions $\mathbf{R}(\omega) \mathbf{b}_{i}+$ $\mathbf{t}=\mathbf{a}_{i}+l_{i} \mathbf{e}_{i}$. Thus, the wire length $l_{i}$ can be expressed as

$$
l_{i}=\left\|\mathbf{R}(\omega) \mathbf{b}_{i}+\mathbf{t}-\mathbf{a}_{i}\right\| .
$$

Let $\delta l_{i}$ and $l_{i}^{0}$ denote the additive random error and the actual value of distance measurement $l_{i}$, respectively. Let $\delta \mathbf{I}=\left(\delta l_{1} \ldots \delta l_{6}\right)^{t}$ and $\mathbf{l}^{0}=\left(l_{1}^{0} \ldots l_{6}^{0}\right)^{t}$. Then, $\mathbf{l}=\mathbf{l}^{0}+\delta \mathbf{l}$.

The wire length errors are assumed to have zero mean value, that is $E\{\delta \mathbf{I}\}=0$, where $E\{\cdot\}$ stands for the expected value operation. We also assume that the wire length errors are uncorrelated with the same variance $\sigma^{2}$. Consequently, their covariance matrix can be expressed as

$$
E\left\{\delta \mathbf{l} \delta \mathbf{l}^{t}\right\}=\sigma^{2} \mathbf{I},
$$


where I denotes the identity matrix.

Then, the linearization of (1) around the measured value of $l_{i}\left(l_{i}^{0}\right)$, and the calculated value of the configuration $\mathbf{q}\left(\mathbf{q}^{0}\right)$ is

$$
l_{i}-l_{i}^{0}=\frac{\partial l_{i}}{\partial \mathbf{q}}\left(\mathbf{q}-\mathbf{q}^{0}\right)=\left(\begin{array}{c}
\left(\mathbf{t}-\mathbf{a}_{i}\right) \times \mathbf{e}_{i} \\
\mathbf{e}_{i}
\end{array}\right)^{t}\left(\mathbf{q}-\mathbf{q}^{0}\right) .
$$

Collecting (3) for the six wires into a single matrix expression, we get

$$
\delta \mathbf{l}=\mathbf{l}-\mathbf{l}^{0}=\mathbf{J}\left(\mathbf{q}-\mathbf{q}^{0}\right)=\mathbf{J} \delta \mathbf{q},
$$

where $\mathbf{J}=\left(\begin{array}{ccc}\left(\mathbf{t}-\mathbf{a}_{1}\right) \times \mathbf{e}_{1} & \cdots & \left(\mathbf{t}-\mathbf{a}_{6}\right) \times \mathbf{e}_{6} \\ \mathbf{e}_{1} & \cdots & \mathbf{e}_{6}\end{array}\right)^{t}$.

Since $E\left\{\delta \mathbf{l} \delta \mathbf{I}^{t}\right\}=\mathbf{J} E\left\{\delta \mathbf{q} \delta \mathbf{q}^{t}\right\} \mathbf{J}^{t}=\sigma^{2} \mathbf{I}$, the covariance matrix for the pose is

$$
E\left\{\delta \mathbf{q} \delta \mathbf{q}^{t}\right\}=\sigma^{2} \mathbf{J}^{-1}\left(\mathbf{J}^{t}\right)^{-1}=\sigma^{2}\left(\mathbf{J}^{t} \mathbf{J}\right)^{-1} .
$$

Note that (5) is well-defined provided that $\mathbf{J}$ is nonsingular. If screw coordinates are used -instead of roll, pitch and yaw angles- the rows of $\mathbf{J}$ would directly be the Plücker coordinates of the wire lines. In our case, it can be checked these rows are the Plücker coordinates of an arrangement of lines equivalent to that of the wire lines. Then, it is possible to characterize the singularities of $\mathbf{J}$ in terms of the geometry of linearly dependent sets of lines [16, p. 274-284]. Nevertheless, in Section V we will use an alternative approach that takes advantage of the 3-2-1 configuration.

Now, since the actual configuration of the platform is contained in the ellipsoid

$$
\left(\mathbf{q}-\mathbf{q}_{0}\right)\left(\mathbf{J}^{t} \mathbf{J}\right)^{-1}\left(\mathbf{q}-\mathbf{q}_{0}\right) \leq 16
$$

with probability 0.99 , it might seem reasonable to use the volume of this ellipsoid (i.e. the square root of the determinant of $\left.\left(\mathbf{J}^{t} \mathbf{J}\right)^{-1}\right)$ as the measurement of this sensitivity, as it is proposed in [6]. Unfortunately, the matrix $\left(\mathbf{J}^{t} \mathbf{J}\right)^{-1}$ does not make physical sense. Actually, its elements are the sum of a quantity of lengthsquared added to a unitless quantity, which leads to what is technically called a nonconmmensurate system [20]. Moreover, a sensitivity measurement based on the volume of (6) cannot be used to compare different wire configurations, contrary to what it is done in [6], because it is not independent from the chosen reference frames.

The above inconveniences motivate the search for a coordinate-free formulation, but first we need to introduce some concepts related to Cayley-Menger determinants and the geometry of tetrahedra.

\section{CAyley-Menger Determinants}

The Cayley-Menger bideterminant of two sequences of $n$ points, $\left[\mathbf{p}_{1}, \ldots, \mathbf{p}_{n}\right]$ and $\left[\mathbf{q}_{1}, \ldots, \mathbf{q}_{n}\right]$, is defined as:

$$
\begin{aligned}
& \mathbf{D}\left(\mathbf{p}_{1}, \ldots, \mathbf{p}_{n} ; \mathbf{q}_{1}, \ldots, \mathbf{q}_{n}\right)=2\left(\frac{-1}{2}\right)^{n} . \\
& .\left|\begin{array}{ccccc}
0 & 1 & 1 & 1 & 1 \\
1 & D\left(\mathbf{p}_{1}, \mathbf{q}_{1}\right) & D\left(\mathbf{p}_{1}, \mathbf{q}_{2}\right) & \cdots & D\left(\mathbf{p}_{1}, \mathbf{q}_{n}\right) \\
1 & D\left(\mathbf{p}_{2}, \mathbf{q}_{1}\right) & D\left(\mathbf{p}_{2}, \mathbf{q}_{2}\right) & \cdots & D\left(\mathbf{p}_{2}, \mathbf{q}_{n}\right) \\
\vdots & \vdots & \vdots & \ddots & \vdots \\
1 & D\left(\mathbf{p}_{n}, \mathbf{q}_{1}\right) & D\left(\mathbf{p}_{n}, \mathbf{q}_{2}\right) & \cdots & D\left(\mathbf{p}_{n}, \mathbf{q}_{n}\right)
\end{array}\right|,
\end{aligned}
$$

where $D\left(\mathbf{p}_{i}, \mathbf{q}_{j}\right)$ denotes the squared distance between the points $\mathbf{p}_{i}$ and $\mathbf{q}_{j}$.

This determinant plays a fundamental role in the so-called "Distance Geometry," a term coined by L. Blumenthal in [1] which refers to the analytical study of the Euclidean geometry in terms of invariants without resorting to artificial coordinate systems. (The constant factor in this definition simplifies the geometric interpretation of these determinants, as will be seen below.) Since in many cases of interest the two sequences of points are the same, it will be convenient to abbreviate $D\left(\mathbf{p}_{1}, \ldots, \mathbf{p}_{n} ; \mathbf{p}_{1}, \ldots, \mathbf{p}_{n}\right)$ by $D\left(\mathbf{p}_{1}, \ldots, \mathbf{p}_{n}\right)$, which is simply called a Cayley-Menger determinant.

To set the background for later developments, we give the geometric interpretation of these determinants for $n=2,3,4$.

For the Cayley-Menger determinants, it can be shown that $D\left(\mathbf{p}_{1}, \ldots, \mathbf{p}_{n}\right)$ is $((n-1) !)^{2}$ times the squared hypervolume of the simplex spanned by the points $\mathbf{p}_{1}, \ldots, \mathbf{p}_{n}$. For further details the reader is referred to [8] and to [4, pp. 126-129].

Hence, for $n=2: D\left(\mathbf{p}_{1}, \mathbf{p}_{2}\right)=d\left(\mathbf{p}_{1}, \mathbf{p}_{2}\right)^{2}$, where $d\left(\mathbf{p}_{1}, \mathbf{p}_{2}\right)$ is the Euclidean distance between $\mathbf{p}_{1}$ and $\mathbf{p}_{2}$. Observe that the use of the symbol $D\left(\mathbf{p}_{i}, \mathbf{p}_{j}\right)$ for both the squared distance from $\mathbf{p}_{1}$ to $\mathbf{p}_{2}$ and their CayleyMenger determinant is consistent.

For $n=3$, if $A$ is the area of the triangle spanned by $\mathbf{p}_{1}, \mathbf{p}_{2}$ and $\mathbf{p}_{3}$, we obtain Herron's formula relating $A$ with the side-lengths:

$$
D\left(\mathbf{p}_{1}, \mathbf{p}_{2}, \mathbf{p}_{3}\right)=4 A^{2} \text {. }
$$

For $n=4$, if $V$ is the volume of the tetrahedron spanned by $\mathbf{p}_{1}, \mathbf{p}_{2}, \mathbf{p}_{3}$, and $\mathbf{p}_{4}$, we obtain Eurler's formula relating $V$ with the edge-lengths:

$$
D\left(\mathbf{p}_{1}, \mathbf{p}_{2}, \mathbf{p}_{3}, \mathbf{p}_{4}\right)=36 V^{2} \text {. }
$$

For the Cayley-Menger bideterminants, it can be shown that, for $n=2$ :

$$
D\left(\mathbf{p}_{1}, \mathbf{p}_{2} ; \mathbf{q}_{1}, \mathbf{q}_{2}\right)=\left(\mathbf{p}_{1}-\mathbf{p}_{2}\right) \cdot\left(\mathbf{q}_{1}-\mathbf{q}_{2}\right),
$$

Since this dot product can be expressed as $d\left(\mathbf{p}_{1}, \mathbf{p}_{2}\right) d\left(\mathbf{q}_{1}, \mathbf{q}_{2}\right) \cos (\boldsymbol{\theta})$, with $\boldsymbol{\theta}$ being the angle 
between the two lines $\mathbf{p}_{1} \mathbf{p}_{2}$ and $\mathbf{q}_{1} \mathbf{q}_{2}$, this yields the following formula for $\cos (\theta)$ in terms of the six inter-point distances:

$$
\cos \theta=\frac{D\left(\mathbf{p}_{1}, \mathbf{p}_{2} ; \mathbf{q}_{1}, \mathbf{q}_{2}\right)}{d\left(\mathbf{p}_{1}, \mathbf{p}_{2}\right) d\left(\mathbf{q}_{1}, \mathbf{q}_{2}\right)}
$$

The reader can easily see that when $\mathbf{p}_{1}=\mathbf{q}_{1}$ this formula reduces to the law of cosines for a triangle, by expanding $D\left(\mathbf{p}_{1}, \mathbf{p}_{2} ; \mathbf{q}_{1}, \mathbf{q}_{2}\right)$ in terms of the involved distances.

Likewise, for $n=3$ it can be shown that:

$$
\begin{aligned}
& D\left(\mathbf{p}_{1}, \mathbf{p}_{2}, \mathbf{p}_{3} ; \mathbf{q}_{1}, \mathbf{q}_{2}, \mathbf{q}_{3}\right)= \\
& \quad\left(\left(\mathbf{p}_{1}-\mathbf{p}_{3}\right) \times\left(\mathbf{p}_{2}-\mathbf{p}_{3}\right)\right) \cdot\left(\left(\mathbf{q}_{1}-\mathbf{q}_{3}\right) \times\left(\mathbf{q}_{2}-\mathbf{q}_{3}\right)\right),
\end{aligned}
$$

The right hand side of this equation can be easily shown to be equal to $2 A_{1} \cdot 2 A_{2} \cdot \cos (\phi)$, where $A_{1}$ and $A_{2}$ are the areas of the triangles $\mathbf{p}_{1}, \mathbf{p}_{2}, \mathbf{p}_{3}$ and $\mathbf{q}_{1}, \mathbf{q}_{2}, \mathbf{q}_{3}$, respectively, and $\phi$ is the dihedral angle between the planes they define. By expressing these areas as Cayley-Menger determinants of the triangles' points, this yields the following formula for the cosine of $\phi$ in terms of inter-point distances:

$$
\cos \phi=\frac{D\left(\mathbf{p}_{1}, \mathbf{p}_{2}, \mathbf{p}_{3} ; \mathbf{q}_{1}, \mathbf{q}_{2}, \mathbf{q}_{3}\right)}{D^{\frac{1}{2}}\left(\mathbf{p}_{1}, \mathbf{p}_{2}, \mathbf{p}_{3}\right) D^{\frac{1}{2}}\left(\mathbf{q}_{1}, \mathbf{q}_{2}, \mathbf{q}_{3}\right)},
$$

which can be regarded as the law of cosines generalized to a tetrahedron when $\mathbf{p}_{2}=\mathbf{q}_{2}$ and $\mathbf{p}_{3}=\mathbf{q}_{3}$ (see [12] for an alternative formulation). Finally, for $n=4$, the bideterminant is equal to the product of two triple products:

$$
\begin{gathered}
D\left(\mathbf{p}_{1}, \mathbf{p}_{2}, \mathbf{p}_{3}, \mathbf{p}_{4} ; \mathbf{q}_{1}, \mathbf{q}_{2}, \mathbf{q}_{3}, \mathbf{q}_{4}\right)= \\
\left|\mathbf{p}_{1}-\mathbf{p}_{4}, \mathbf{p}_{2}-\mathbf{p}_{4}, \mathbf{p}_{3}-\mathbf{p}_{4}\right| \cdot\left|\mathbf{q}_{1}-\mathbf{q}_{4}, \mathbf{q}_{2}-\mathbf{q}_{4}, \mathbf{q}_{3}-\mathbf{q}_{4}\right|,
\end{gathered}
$$

and hence, it can be interpreted as 36 times the product of the volumes of the two tetrahedra defined by $\mathbf{p}_{1}, \ldots, \mathbf{p}_{4}$ and $\mathbf{q}_{1}, \ldots, \mathbf{q}_{4}$.

\section{A COORDINATE-FREE FORMULATION FOR TRILATERATION}

Given three points in space, say $\mathbf{p}_{1}, \mathbf{p}_{2}$, and $\mathbf{p}_{3}$, the trilateration problem consists in finding the location of another point, say $\mathbf{p}_{4}$, whose distance to these three points is known. According to Figure 2, using barycentric coordinates [3, pp. 216-221], the location of the orthogonal projection of $\mathbf{p}_{4}$, say $\mathbf{p}$, onto the plane defined by $\mathbf{p}_{1}, \mathbf{p}_{2}$ and $\mathbf{p}_{3}$ (hereafter the base plane) can be expressed as

$$
\mathbf{p}=\frac{A_{1} \mathbf{p}_{1}+A_{2} \mathbf{p}_{2}+A_{3} \mathbf{p}_{3}}{A_{b}}
$$

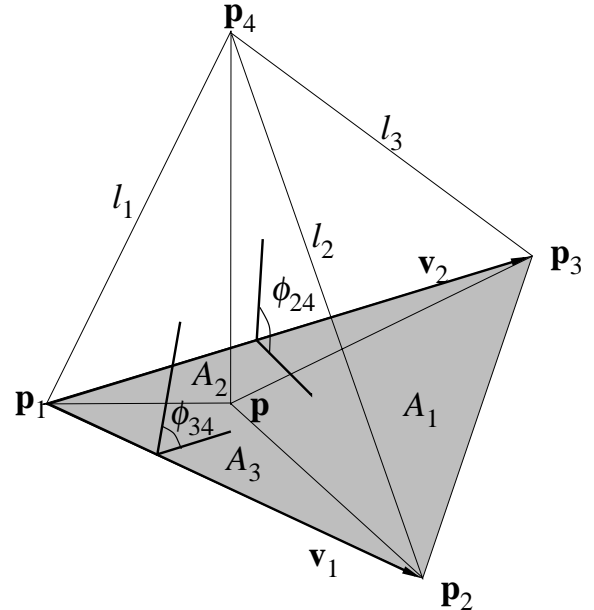

Fig. 2. Barycentric coordinates of the projection of $\mathbf{p}_{4}$ onto the plane defined by $\mathbf{p}_{1}, \mathbf{p}_{2}$, and $\mathbf{p}_{3}$.

where $A_{1}, A_{2}$ and $A_{3}$ are the signed areas ${ }^{1}$ of the triangles $\mathbf{p}_{2} \mathbf{p}_{3} \mathbf{p}, \mathbf{p}_{3} \mathbf{p}_{1} \mathbf{p}$ and $\mathbf{p}_{1} \mathbf{p}_{2} \mathbf{p}$, respectively, and $A_{b}$ is the area of the triangle $\mathbf{p}_{1} \mathbf{p}_{2} \mathbf{p}_{3}$. Alternatively,

$$
\mathbf{p}=\mathbf{p}_{1}+\frac{A_{2}}{A_{b}} \mathbf{v}_{1}+\frac{A_{3}}{A_{b}} \mathbf{v}_{2},
$$

where $\mathbf{v}_{1}=\mathbf{p}_{2}-\mathbf{p}_{1}$ and $\mathbf{v}_{2}=\mathbf{p}_{3}-\mathbf{p}_{1}$.

The values $A_{i}$ can be obtained by projecting the areas of the triangles coincident in $\mathbf{p}_{4}$, onto the base plane. Hence, using Equation (7):

$$
\begin{aligned}
& \frac{A_{2}}{A_{b}}=\sqrt{\frac{D\left(\mathbf{p}_{1}, \mathbf{p}_{3}, \mathbf{p}_{4}\right)}{D\left(\mathbf{p}_{1}, \mathbf{p}_{2}, \mathbf{p}_{3}\right)}} \cos \left(\phi_{24}\right), \\
& \frac{A_{3}}{A_{b}}=\sqrt{\frac{D\left(\mathbf{p}_{1}, \mathbf{p}_{2}, \mathbf{p}_{4}\right)}{D\left(\mathbf{p}_{1}, \mathbf{p}_{2}, \mathbf{p}_{3}\right)}} \cos \left(\phi_{34}\right),
\end{aligned}
$$

where $\phi_{24}$ and $\phi_{34}$ are the dihedral angles indicated in Figure 2. Now, using Equation (9) we can write:

$$
\mathbf{p}=\mathbf{p}_{1}+k_{1} \mathbf{v}_{1}+k_{2} \mathbf{v}_{2},
$$

where

$$
\begin{gathered}
k_{1}=-\frac{D\left(\mathbf{p}_{1}, \mathbf{p}_{2}, \mathbf{p}_{3} ; \mathbf{p}_{1}, \mathbf{p}_{3}, \mathbf{p}_{4}\right)}{D\left(\mathbf{p}_{1}, \mathbf{p}_{2}, \mathbf{p}_{3}\right)}, \\
k_{2}=\frac{D\left(\mathbf{p}_{1}, \mathbf{p}_{2}, \mathbf{p}_{3} ; \mathbf{p}_{1}, \mathbf{p}_{2}, \mathbf{p}_{4}\right)}{D\left(\mathbf{p}_{1}, \mathbf{p}_{2}, \mathbf{p}_{3}\right)} .
\end{gathered}
$$

Finally, $\mathbf{p}_{4}$ can be obtained as:

$$
\mathbf{p}_{4}=\mathbf{p} \pm k_{3}\left(\mathbf{v}_{1} \times \mathbf{v}_{2}\right),
$$

where the \pm sign accounts for the two mirror symmetric solutions of $\mathbf{p}_{4}$ with respect to the base plane, and $k_{3}$ is equal to the height $h$ of the tetrahedron divided by the

\footnotetext{
${ }^{1}$ For a triangle pqr with area $A$, the signed area is defined as $+A$ (respectively $-A$ ) if the point $\mathbf{q}$ is to the right (respectively to the left) of the line pr, when going from $\mathbf{p}$ to $\mathbf{r}$.
} 
norm of $\mathbf{v}_{1} \times \mathbf{v}_{2}$. Since the volume of the tetrahedron is $\frac{1}{3} A_{b} h$, using Equations (7) and (8) we can write:

$$
h=\sqrt{\frac{D\left(\mathbf{p}_{1}, \mathbf{p}_{2}, \mathbf{p}_{3}, \mathbf{p}_{4}\right)}{D\left(\mathbf{p}_{1}, \mathbf{p}_{2}, \mathbf{p}_{3}\right)}} .
$$

Moreover, since $\left\|\mathbf{v}_{1} \times \mathbf{v}_{2}\right\|$ is twice the area of the triangle $\mathbf{p}_{1}, \mathbf{p}_{2}, \mathbf{p}_{3}$,

$$
\left\|\mathbf{v}_{1} \times \mathbf{v}_{2}\right\|=\sqrt{D\left(\mathbf{p}_{1}, \mathbf{p}_{2}, \mathbf{p}_{3}\right)},
$$

one concludes that

$$
k_{3}=\frac{\sqrt{D\left(\mathbf{p}_{1}, \mathbf{p}_{2}, \mathbf{p}_{3}, \mathbf{p}_{4}\right)}}{D\left(\mathbf{p}_{1}, \mathbf{p}_{2}, \mathbf{p}_{3}\right)} .
$$

Hence, the final expression for $\mathbf{p}_{4}$ is:

$$
\begin{aligned}
\mathbf{p}_{4}=\frac{1}{D\left(\mathbf{p}_{1}, \mathbf{p}_{2}, \mathbf{p}_{3}\right)} \cdot( & -D\left(\mathbf{p}_{1}, \mathbf{p}_{2}, \mathbf{p}_{3} ; \mathbf{p}_{1}, \mathbf{p}_{3}, \mathbf{p}_{4}\right) \cdot \mathbf{v}_{1} \\
& +D\left(\mathbf{p}_{1}, \mathbf{p}_{2}, \mathbf{p}_{3} ; \mathbf{p}_{1}, \mathbf{p}_{2}, \mathbf{p}_{4}\right) \cdot \mathbf{v}_{2} \\
& \left. \pm \sqrt{D\left(\mathbf{p}_{1}, \mathbf{p}_{2}, \mathbf{p}_{3}, \mathbf{p}_{4}\right)} \cdot\left(\mathbf{v}_{1} \times \mathbf{v}_{2}\right)\right) .
\end{aligned}
$$

Most solutions for trilateration are expressed according to a specific coordinate frame. For example, in [6], the $\mathrm{XY}$ plane is the plane defined by $\mathbf{p}_{1}, \mathbf{p}_{2}$ and $\mathbf{p}_{3}$, the $\mathrm{X}$ axis is defined by the line containing $\mathbf{p}_{1}$ and $\mathbf{p}_{2}$ and the origin of the frame is located at $\mathbf{p}_{1}$. The formulation given here is coordinate-free because it only deals with inter-point distances. Hence, its numerical conditioning is independent from the chosen reference frames.

Although the formulations presented in [13] and [2] can also be classified as coordinate-free, the one presented here is mathematically more tractable because all terms are determinants with geometric meaning. Thus, it accommodates a more thorough investigation of the effects caused by wire length errors and singularities, as shown below. Moreover, its accurate evaluation using floating point arithmetic can also be simplified because it boils down to the accurate evaluation of Cayley-Menger determinants (the reader is addressed to [11] for considerations on this point).

For small wire length errors, the error in the location of $\mathbf{p}_{4}$ can be well approximated by retaining the terms up to the second-order partial derivatives in the Taylor expansion of Equation (10), that is

$$
\delta \mathbf{p}_{4}=\mathbf{p}_{4}-\mathbf{p}_{4}^{0}=\sum_{i=1}^{3} \frac{\partial \mathbf{p}_{4}}{\partial l_{i}} \delta l_{i}+\frac{1}{2} \sum_{i=1}^{3} \sum_{j=1}^{3} \frac{\partial^{2} \mathbf{p}_{4}}{\partial l_{i} \partial l_{j}} \delta l_{i} \delta l_{j} .
$$

Then, the expected value of the random error in $\mathbf{p}_{4}$, i.e. the bias error, is

$$
E\left\{\delta \mathbf{p}_{4}\right\}=\frac{1}{2} \sum_{i=1}^{3} \sum_{j=1}^{3} \frac{\partial^{2} \mathbf{b}_{1}}{\partial l_{i} \partial l_{j}} E\left\{\delta l_{i} \delta l_{j}\right\} .
$$

In other words, although the noise in the length measurements is assumed to have zero mean value, the expected value of the estimation error obtained by trilateration does not equal zero due to nonlinearities.

Then, using (2),

$$
E\left\{\delta \mathbf{p}_{4}\right\}=\frac{\sigma^{2}}{2}\left(\frac{\partial^{2} \mathbf{p}_{4}}{\partial l_{1}^{2}}+\frac{\partial^{2} \mathbf{p}_{4}}{\partial l_{2}^{2}}+\frac{\partial^{2} \mathbf{p}_{4}}{\partial l_{3}^{2}}\right) .
$$

Finally, substituting (10),

$$
E\left\{\delta \mathbf{p}_{4}\right\}=\frac{\sigma^{2}}{2}\left(\nabla^{2} k_{1} \mathbf{v}_{1}+\nabla^{2} k_{2} \mathbf{v}_{2}+\nabla^{2} k_{3}\left(\mathbf{v}_{1} \times \mathbf{v}_{2}\right)\right),
$$

where $\nabla^{2} k_{i}=\frac{\partial^{2} k_{i}}{\partial l_{1}^{2}}+\frac{\partial^{2} k_{i}}{\partial l_{2}^{2}}+\frac{\partial^{2} k_{i}}{\partial l_{3}^{2}}$.

The trilateration bias vector error was already examined in [13], where it was shown that its projection onto the plane defined by $\mathbf{p}_{1}, \mathbf{p}_{2}$ and $\mathbf{p}_{3}$ can be neglected. Using our formulation, this fact can simply be stated as follows:

$$
E\left\{\delta \mathbf{p}_{4}\right\} \simeq \frac{\sigma^{2}}{2} \nabla^{2} k_{3}\left(\mathbf{v}_{1} \times \mathbf{v}_{2}\right) .
$$

It can be checked that, as a consequence of this error, when the unknown point is moved on a plane parallel to that of the three known points, the estimation will erroneously indicate that it ascends and descends when it approaches to, and goes away from the center of the three known points, respectively. Fortunately, since this is a systematic error, it can be compensated by proper calibration.

The covariance matrix, $\mathbf{C}$, of the position estimate error $\delta \mathbf{p}_{4}$ can now be evaluated as

$$
\begin{aligned}
\mathbf{C} & =E\left\{\left[\delta \mathbf{p}_{4}-E\left\{\delta \mathbf{p}_{4}\right\}\right]\left[\delta \mathbf{p}_{4}-E\left\{\delta \mathbf{p}_{4}\right\}\right]^{t}\right\} \\
& =E\left\{\delta \mathbf{p}_{4} \delta \mathbf{p}_{4}^{t}\right\}-E\left\{\delta \mathbf{p}_{4}\right\} E\left\{\delta \mathbf{p}_{4}^{t}\right\} .
\end{aligned}
$$

Since the second term contains the multiplicative factor $\sigma^{4}$, it can be neglected for small wire length errors. Thus,

$$
\mathbf{C} \simeq E\left\{\frac{\partial \mathbf{p}_{4}}{\partial \mathbf{r}} \delta \mathbf{r} \delta \mathbf{r}^{t}\left(\frac{\partial \mathbf{p}_{4}}{\partial \mathbf{r}}\right)^{t}\right\}=\sigma^{2} \frac{\partial \mathbf{p}_{4}}{\partial \mathbf{r}}\left(\frac{\partial \mathbf{p}_{4}}{\partial \mathbf{r}}\right)^{t},
$$

where $\mathbf{r}=\left(l_{1}, l_{2}, l_{3}\right)^{t}$.

Explicit expressions for all the partial derivatives appearing in this section, in terms of Cayley-Menger bideterminants, can be found in [19], where the error in the location of $\mathbf{p}_{4}$ due to the errors in the location of $\mathbf{p}_{1}, \mathbf{p}_{2}$ and $\mathbf{p}_{3}$ is also analyzed.

\section{FORWARD KINEMATICS AND SINGULARITIES}

The direct kinematics of the 3-2-1 configuration can be solved by three consecutive trilateration operations. Indeed, according to Fig. 3a, given $l_{1}, l_{2}$, and $l_{3}$, there are two possible mirror locations for $\mathbf{b}_{1}$ with respect to the plane defined by $\mathbf{a}_{1}, \mathbf{a}_{2}$, and $\mathbf{a}_{3}$ (Figure $3 b$ ). Once one of these two solutions for $\mathbf{b}_{1}$ is chosen, $\mathbf{a}_{4}, \mathbf{a}_{5}$, 


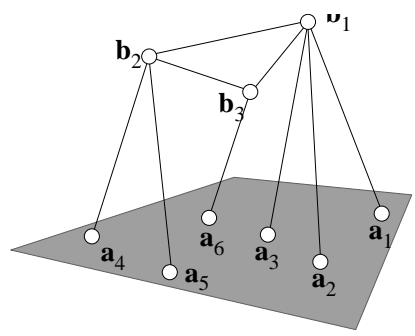

(a)

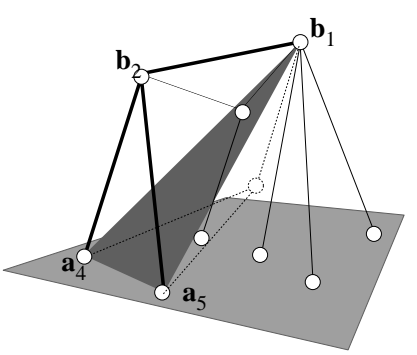

(c)

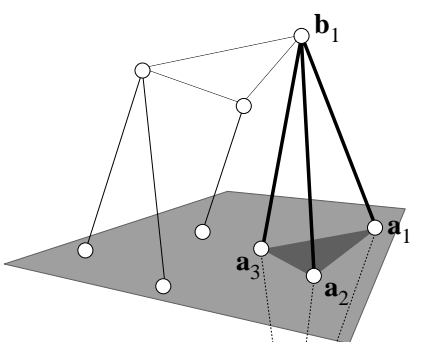

(b)

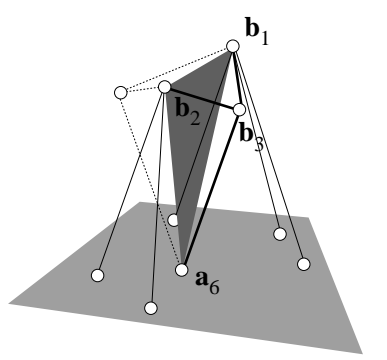

(d)
Fig. 3. (a) General kinematic model of a 3-2-1 tracking system. There are up to 8 possible solutions for the configuration of the platform compatible with a set of wire lengths due to the two possible solutions for the location of $\mathbf{b}_{1}(\mathbf{b}), \mathbf{b}_{2}(\mathbf{c})$, and $\mathbf{b}_{3}(\mathbf{d})$.

$\mathbf{b}_{1}$ and $\mathbf{b}_{2}$ define another tetrahedron with known edge lengths. Again, there are two possible mirror locations for $\mathbf{b}_{2}$, in this case with respect to the plane defined by $\mathbf{a}_{4}, \mathbf{a}_{5}$, and $\mathbf{b}_{1}$ (Figure 3c). Finally, after choosing one of the two solutions, $\mathbf{a}_{6}, \mathbf{b}_{1}, \mathbf{b}_{2}$, and $\mathbf{b}_{3}$ define another tetrahedron with known edge lengths. In this case there are two mirror possible locations for $\mathbf{b}_{3}$ with respect to the plane defined by $\mathbf{a}_{6}, \mathbf{b}_{1}$ and $\mathbf{b}_{2}$ (Figure $3 \mathrm{~d}$ ).

Once points $\mathbf{b}_{1}, \mathbf{b}_{2}$, and $\mathbf{b}_{3}$ have been located, they can be used to define a reference frame on the moving object. For example, the $x$-axis can be defined by the direction given by $\left(\mathbf{b}_{2}-\mathbf{b}_{1}\right)$, the $y$-axis can be chosen orthogonal to the plane defined by $\left(\mathbf{b}_{2}-\mathbf{b}_{1}\right)$ and $\left(\mathbf{b}_{3}-\right.$ $\mathbf{b}_{1}$ ), and the $z$-axis can be obtained to give a Cartesian reference frame. These vectors form a set of orthogonal basis vectors whose directions are known relative to both the fixed and the moving reference frames. This completely solves the forward kinematics of the 3-21 configuration without evaluating any trigonometric function.

There are certain singular sets of wire lengths yielding less than eight solutions. For example, according to Equation (10), the first trilateration operation will yield only one solution if, and only if, $D\left(\mathbf{a}_{1}, \mathbf{a}_{2}, \mathbf{a}_{3}, \mathbf{b}_{1}\right)=0$, i.e., if $\mathbf{a}_{1}, \mathbf{a}_{2}, \mathbf{a}_{3}$, and $\mathbf{b}_{1}$ lie on the same plane. Also, the result is undefined if, and only if, $D\left(\mathbf{a}_{1}, \mathbf{a}_{2}, \mathbf{a}_{3}\right)=0$, i.e., if $\mathbf{a}_{1}, \mathbf{a}_{2}$, and $\mathbf{a}_{3}$ are aligned. Since $D\left(\mathbf{a}_{1}, \mathbf{a}_{2}, \mathbf{a}_{3}\right)=$ 0 implies $D\left(\mathbf{a}_{1}, \mathbf{a}_{2}, \mathbf{a}_{3}, \mathbf{b}_{1}\right)=0$, this latter condition encompasses all singularities for the first trilateration. This reasoning can be repeated for the other two trilateration operations concluding that, if the platform is in a configuration in which $D\left(\mathbf{a}_{1}, \mathbf{a}_{2}, \mathbf{a}_{3}, \mathbf{b}_{1}\right)=0$, $D\left(\mathbf{a}_{4}, \mathbf{a}_{5}, \mathbf{b}_{1}, \mathbf{b}_{2}\right)=0$, or $D\left(\mathbf{a}_{6}, \mathbf{b}_{1}, \mathbf{b}_{2}, \mathbf{b}_{3}\right)=0$, the tracking system is in a singularity. In other words, these three equations fully characterize all the singularities. Each of them defines a variety of dimension 5 that divides the configuration space of the platform, i.e. $\mathfrak{R}^{3} \times S O(3)$, into two half-spaces. Then, they lead to a partition of this configuration space into 8 regions with congruent signs for the corresponding 3 determinants. During normal operation, the tracking system should work in one of these regions without getting out of it to avoid ambiguities. Nevertheless, either by assuming continuity in the velocity vector or by using an extra sensor such as an inclinometer, it is possible to move from one of these regions to an adjacent one without ambiguities.

\section{CONCLUSIONS}

The formulation presented in this paper is currently being validated using the hardware implementation described in [17].

At the beginning of a measuring cycle, the pose of the platform is assumed to be known. This requires deciding the orientation of three tetrahedra which can be done by visual inspection or using an extra sensor. When traversing a singularity, an ambiguity arises. Nevertheless, since infinity accelerations are not physically feasible, an algorithm can be designed to track a unique solution based on the continuity of the velocity vector. Moreover, the measurements along a trajectory are not statistically uncorrelated so that they should be jointly smoothed during tracking to improve accuracy using, for example, a Kalman filter. The probabilistic uncertainty model given in this paper is of relevance to this end. For example, the characterization of the bias error must not be ignored at this point and it has to be suitably anticipated in this filter. These issues concentrate our current efforts.

\section{ACKNOWLEDGEMENTS}

This work has been partially supported by the Italian-Spanish Bilateral CSIC-CNR Cooperation Committee and the Spanish CICYT under contract TIC2000-0696.

\section{REFERENCES}

[1] L.M. Blumenthal, Theory and Applications of Distance Geometry, Oxford University Press, 1953.

[2] H. Bruyninckx, "Forward kinematics for Hunt-Primrose parallel manipulators," Mechanism and Machine Theory, Vol. 34, pp. 657-664, 1999. 
[3] H.S.M. Coxeter, Introduction to Geometry, John Wiley and Sons Inc., New York, 1989.

[4] G.M. Crippen and T. Havel, Distance Geometry and Molecular Conformation. John Wiley and Sons Inc., New York, 1988.

[5] D. Eberly, "Finding the intersection of three spheres," comp.graphics.algorithms newsgroup, 1996.

[6] Z. Geng and L.S. Haynes, "A 3-2-1 kinematic configuration of a Stewart platform and its application to six degree of freedom pose measurements," Robotics and Computer-Integrated Manufacturing, Vol. 11, No. 1, pp. 23-34, 1994.

[7] M. Griffis and J. Duffy, "A forward displacement analysis of a class of Stewart platforms," Journal of Robotic Systems, Vol. 6, No. 6, pp. 703-720, 1989.

[8] T. Havel, "Some examples of the use of distances as coordinates in Euclidean geometry," Journal of Symbolic Computation, Vol. 11, pp. 579-593, 1991.

[9] K.H. Hunt and E.J.F. Primrose, "Assembly configurations of some in-parallel-actuated manipulators," Mechanism and Machine Theory, Vol. 28, No. 1, pp. 31-42, 1993.

[10] J.W. Jeong, S.H. Kim, and Y.K. Kwak, "Kinematics and workspace analysis of a parallel wire mechanism for measuring a robot pose," Mechanism and Machine Theory, Vol. 34, No. 6, pp. 825-841, 1999.

[11] W. Kahan, "What has the volume of a tetrahedron to do with computer programming languages," Web document available at www.cs.berkeley.edu/ ${ }^{\sim}$ wkahan/VtetLang.pdf

[12] J.R. Lee, "The law of cosines in a tetrahedron," J. Korea Soc. Math. Ed. Ser. B: Pure Appl. Math., No. 4, pp. 1-6, 1997.

[13] D.E. Manolakis, "Efficient solution and performance analysis of 3-D position estimation by trilateration," IEEE Trans. on Aerospace and Electronic Systems, Vol. 32, No. 4, pp. 1239-1248, 1996.

[14] P. Nanua and K.J. Waldron, "Direct kinematics solution of a special parallel robot structure," Proc. RoManSy 8 , pp. 134-142, 1990.

[15] P. Nanua, K.J. Waldron, and V. Murthy, "Direct kinematic solution of a Stewart platform," IEEE Trans. on Robotics and Automation, Vol. 6, No. 4, pp. 438-444, 1990.

[16] J.M. McCarthy, Geometric Design of Linkages, Springer-Verlag, New York, 2000.

[17] E. Ottaviano, M. Ceccarelli, M. Totti, and C. AvilaCarrasco, "CATRASYS (Cassino Tracking System): A wire system for experimental evaluation of robot workspace," Journal of Robotics and Mechatronics, Vol. 14, No. 1, pp. 78-87, 2002.

[18] V. Parenti-Castelli and C. Innocenti, "Direct displacement analysis for some classes of spatial parallel machanisms," Proc. RoManSy 8, pp. 126-133, 1990.

[19] L. Ros and F. Thomas, "Revisiting trilateration," In preparation.

[20] E.M. Schwartz, Algebraic Properties of Noncommensurate Systems and their Applications in Robotics, Ph.D. Dissertation, University of Florida, 1999. 